\title{
Modulators of endothelial cell filopodia
}

\section{PECAM-1 joins the club}

Horace M. DeLisser

Pulmonary, Allergy and Critical Care Division; Department of Medicine; University of Pennsylvania School of Medicine, Philadelphia, PA USA

Key words: angiogenesis, endothelial cells, filopodia, PECAM-1, SHP-2

Submitted: $07 / 14 / 10$

Accepted: 09/10/10

DOI: $10.4161 /$ cam.5.1.13575

Correspondence to: Horace M. DeLisser; Email: delisser@mail.med.upenn.edu
$\mathrm{F}^{\mathrm{s}}$ ilopodia are an important feature of actively motile cells, probing the pericellular environment for chemotactic factors and other molecular cues that enable and direct the movement of the cell. They also act as points of attachment to the extracellular matrix for the cell, generating tension that may act to pull the cell forward and/or stabilize the cell as it moves. Endothelial cell motility is a critical aspect of angiogenesis, but only a limited number of molecules have been identified as specific regulators of endothelial cell filopodia. Recent reports, however, provide evidence for the involvement of PECAM-1, an endothelial cell adhesion and signaling molecule, in the formation of endothelial cell filopodia. This Commentary \& View will focus on these studies and their suggestion that at least two PECAM-1regulated pathways are involved in the processes that enable filopodial protrusions by endothelial cells. Developing a more complete understanding of the role of PECAM-1 in mediating various endothelial cell activities, such as the extension of filopodia, will be essential for exploiting the therapeutic potential of targeting PECAM-1.

The regulated polymerization of actin filaments result in two morphologically distinct protrusive structures, lamellipodia and filopodia, at the leading edge of motile cells. ${ }^{1}$ The lamellipodia are thin (thickness 0.1-0.2 $\mu \mathrm{m}$ ) fan-like projections composed of a branched network of actin., ${ }^{2,3}$ In contrast, filopodia are slender, fingerlike extensions (diameter $0.1-0.3 \mu \mathrm{m}$ ), often emanating from lamellipodia, made up of tight bundles of actin filaments. With respect to directed cell migration, filopodia are particularly important, probing and sensing the pericellular environment for chemotactic and other molecular cues in the extracellular matrix (ECM) that guide the direction and movement of the cell. They also act as points of attachment to the ECM for the cell, generating tension that may act to pull the cell forward and/or stabilize the cell as it moves. Given these roles, it is not surprising that filopodia contain diverse receptors for ECM proteins and an array of signaling molecules.

The formation of new vessels typically involves the initial outward proliferation and migration of endothelial cells (ECs) from a pre-existing vasculature. ${ }^{6,7}$ The tips of these angiogenic sprouts are made up of highly polarized ECs, characterized by the presence of numerous long filopodia that probe the environment, directing the cell toward angiogenic factors. ${ }^{8}$ Surprisingly, our understanding of the processes that regulate the formation of these cellular protrusions in ECs is still incomplete. Of note, only a limited number of molecules have been identified as specifically involved in the formation of endothelial filopodia. These include VEGF receptors and the neuroplins. ${ }^{9,10}$ Recent reports from our group, however, provide evidence of a role for PECAM-1 as a regulator of filopodia formation in ECs. ${ }^{11,12}$

PECAM-1 is a vascular-associated molecule of the Ig superfamily expressed on leukocytes and platelets as well as ECs, where it is enriched at intercellular junctions. ${ }^{13-16}$ PECAM-1 consists of a 574 amino acid extracellular region organized 
into six cystine linked Ig-like domains, a 19 amino acid transmembrane domain and a 118 amino acid cytoplasmic tail. ${ }^{17} \mathrm{It}$ was initially identified as an adhesion molecule capable of binding interactions with itself or with a number of non-PECAM-1 molecules, including heparin containing proteoglycans. ${ }^{18-22}$ However, subsequent studies led to the recognition that PECAM-1 also participates in intracellular signaling. ${ }^{13-16}$ Although it does not have any intrinsic catalytic activity, the cytoplasmic domain of PECAM-1 contains two tyrosine residues (Y663 and Y686) that each fall within a conserved signaling sequence known as the immunoreceptor tyrosine-based inhibitory motif (ITIM). ${ }^{23}$ Phosphorylation of these two tyrosine residues in PECAM-1 creates docking sites for the binding and activation of several cytosolic signaling molecules containing src homology-2 (SH2) domains. Included among the SH2-containing molecules that associate with PECAM-1 are the protein tyrosine phosphatases, SHP-1 and SHP-2, ${ }^{24-31}$ the inosital phosphatase, SHIP ${ }^{31}$ and phospholipase C- $\gamma .{ }^{31}$ PECAM-1 may also associate with phosphoinositide 3-kinase ${ }^{32}$ and $\beta$ - or $\gamma$-catenin. ${ }^{33,34}$ The ability of PECAM-1 to bind to these various cytosolic molecules enables it to potentially modulate the activity of a number of intracellular signaling pathways.

With respect to its endothelial cell functions, PECAM-1 regulates leukocyte transendothelial migration, ${ }^{35}$ protects against endotoxic and apoptotic stresses ${ }^{36,37}$ and contributes to the molecular sensing of fluid sheer stress. ${ }^{16,38}$ PECAM-1 is also involved in angiogenesis. The initial evidence for this came from studies demonstrating that anti-PECAM-1 antibodies inhibit corneal neovascularization induced by angiogenic implants, ${ }^{39}$ vascularization of subcutaneous Matrigel plugs $^{39}$ and tumor angiogenesis. ${ }^{40,41}$ Subsequent studies in PECAM-1-null mice ${ }^{42}$ have helped to confirm and further refine our understanding of the involvement of PECAM-1 in blood vessel formation. PECAM-1-deficient mice are viable, suggesting that vascular development is sufficiently preserved in the absence of PECAM-1 to permit adequate embryogenesis. However, the angiogenic response in a model of foreign body-induced chronic inflammation ${ }^{43}$ and the vascularization of subcutaneous Matrigel implants and subcutaneous tumors are inhibited in PECAM-1-null mice. ${ }^{11}$ In addition, post-natal lung development (a process dependent on angiogenesis) and the initial post-natal vascularization of the murine retina are impaired in PECAM-1-deficient mice. $^{11,44}$ Significantly, the vascular pattern and density in the eyes and lungs are similar in wild type and PECAM-1-null adult mice (unpublished observations), suggesting that the loss of PECAM-1 delays, but does not prevent the eventual development of the retinal or pulmonary vasculature. Together, these data implicate PECAM-1 in pathological angiogenesis, as well as in post-natal vascular developmental processes, such as those occurring in the eyes and lungs.

One of the mechanisms of PECAM1 's involvement in blood vessel formation appears to be an ability to stimulate endothelial cell motility. ${ }^{11,41,45-47}$ Support for this conclusion comes from studies which have shown that anti-PECAM-1 antibodies inhibit the migration of murine and human ECs, ${ }^{11,41}$ endothelial cells from PECAM-1-deficient mice are less motile compared to their wild-type counterparts $^{11,46}$ and the expression of PECAM-1 in non-PECAM-1 expressing cells enhances cell motility. ${ }^{12,41,45-47}$ This activity in stimulating cell motility appears to involve the dephosphorylation of paxillin by the SHP-2 phosphatase. $^{12,47}$

Its involvement in angiogenesis and endothelial cell motility led to an exploration of whether PECAM-1 might also play a role in the formation of endothelial cell filopodia. ${ }^{11}$ Under various conditions it was observed that filopodia were more numerous and/or longer in length in (1) wild type versus PECAM-1-null murine ECs, (2) control HUVEC compared to HUVEC treated with PECAM-1 siRNA and (3) cell transfectants expressing human PECAM-1 versus non-transfected controls. The significance of these in vitro data was confirmed by the finding that the length and number of filopodial extensions from ECs at the leading edge of the developing retinal vascular plexus were suppressed in PECAM-1-null mice. ${ }^{11}$ The concentration of PECAM-1 in the tips of endothelial filopodia (Fig. 1, unpublished observation) is similar to what has been reported for VEGFR-2 (reviewed in ref. 9) and is consistent with a role for PECAM-1 in the formation of these cellular protrusions. PECAM-1 thus represents a new addition to a relative short list of molecules that have been identified as specific regulators of endothelial filopodia formation.

These data may help in part to explain the vascular phenotype of the PECAM-1null mice. ${ }^{42}$ If PECAM-1 is understood as increasing both the length and number of endothelial filopodia, then its presence enables the endothelial cell to more quickly and/or more efficiently assess the pericellular matrix environment for chemotactic or other pro-migratory factors. In this way, rather than being essential for cell migration, PECAM-1 facilitates the process by promoting the efficiency of endothelial cell motility. This might account for why the loss of PECAM-1 does not compromise the formation of the vasculature in the embryo, and delays, but does not arrest, post-natal vascular development in the eyes and lungs.

Although the specific mechanisms by which PECAM-1 promotes the formation of filopodia remain to be determined and are the subject of ongoing studies, both published and unpublished data are suggestive of some possible processes. First, PECAM-1 antibodies that block heterophilic (non-PECAM-1) ligand binding interactions inhibit filopodia formation by subconfluent HUVEC. ${ }^{11}$ This suggests that in the context of an angiogenic, motile endothelial cell, PECAM-1 heterophilic binding interactions with matrix proteins (e.g., proteoglycans) may transduce signals that activate the formation of the bundled, parallel arrays of actin that provide structural support for the filopodia.

Second, the expression of PECAM-1 in cellular transfectants stimulates the formation of filopodial extensions and wound-induced migration, processes that are both associated with increased ERK activation. ${ }^{12}$ These phenomena are inhibited by mutations of Y663 and Y686 in the cytoplasmic domain that lead to a loss of the ability of PECAM-1 to bind SHP- $2 .{ }^{47}$ They are also suppressed by molecular or pharmacological inhibition of the 


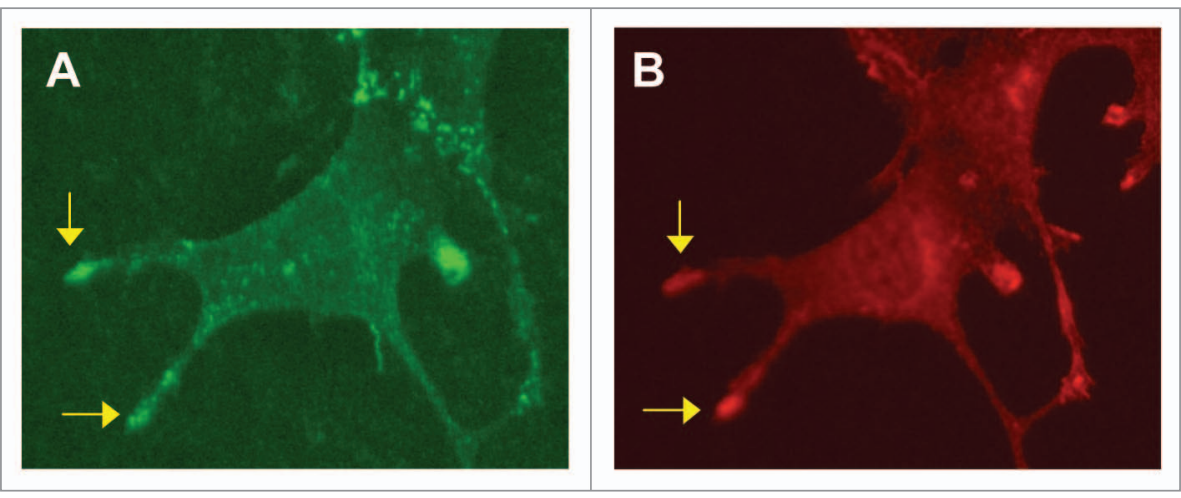

Figure 1. Concentration of PECAM-1 in endothelial filopodia. Shown is an endothelial cell that was immunofluorescently stained for PECAM-1 (A) and VEGFR-2 (B). PECAM-1 and VEGFR-2 are noted to concentrate in the tips of selected filopodia (arrows).

catalytic activity of the SHP-2 phosphatase. ${ }^{2}$ Both cell motility and filopodia are downstream consequences of ERK activation. ${ }^{48,49}$ Further, SHP-2 dephosphorylation of paxillin, with the subsequent activation of src kinase, is one mechanism for the activation of ERK. ${ }^{50,51}$ Our data therefore suggest that the stimulation of filopodia formation mediated by PECAM-1 involves SHP-2-mediated activation of ERK.

Lastly, the presence of PECAM-1 increases the expression of $\mathrm{Cdc} 42$ in murine ECs and in PECAM-1-expressing transfectants. ${ }^{11}$ This is not surprising given the abundance of data linking this Rho-GTPAse to the formation of filopodia. ${ }^{51-54}$ It is important to note, however, that PECAM-1 is expressed at persistently high levels on $\mathrm{ECs}^{17}$ and thus acute increases in PECAM-1 levels are unlikely to directly mediate an increase in Cdc42 expression during angiogenesis. This therefore suggests that if PECAM-1 regulates Cdc42 expression during angiogenesis, this activity involves changes in the activational state PECAM-1 and/or its interaction with other molecules involved in the regulation of Cdc42. In addition, although disturbance of the PECAM-1SHP-2 interaction suppresses PECAM-1dependent filopodia formation, this does not alter the expression of Cdc42 (unpublished data). Together, these data suggest that (i) the influence of PECAM-1 on Rho GTPases in filopodia formation is likely to involve or impact more than just changes in the expression levels of Cdc 42 and (ii) filopodia formation mediated by PECAM-1 includes pathways that are dependent as well as independent of SHP2-regulated ERK activation.

The data generated to date are admittedly descriptive in a number of respects, and the mechanisms of PECAM-1's activity as a regulator of endothelial filopodia are still being defined. However, studies of PECAM-1-transfectants suggest a dynamic interaction between PECAM-1 and SHP-2 in which the level of PECAM-1 tyrosine phosphorylation, and thus SHP-2 binding, are regulated by bound, catalytically-active SHP-2. ${ }^{12}$ Based on these data, along with what was noted above, we suggest the following working model for the involvement of PECAM-1 in the formation of filopodia (Fig. 2). In the setting of post-natal developmental angiogenesis, or during certain forms of pathological angiogenesis, PECAM-1 on angiogenic and/or motile endothelial cells is tyrosine phosphorylated. These phosphorylation events occur either in response to angiogenic growth factor stimulation or are a result of the binding of PECAM-1 to matrix proteins elaborated in the angiogenic context. PECAM-1 phosphorylation subsequently induces the binding of SHP-2 to PECAM-1 and thus its recruitment to the cell membrane. We propose that this binding interaction with PECAM-1 activates the phosphatase activity of SHP-2, with the activated SHP-2 dephosphorylating the PECAM-1 molecule to which it is bound. This leads to the release of SHP-2 from PECAM-1. The liberated but now membrane localized SHP-2 in turn targets paxillin, dephosphorylating it, to activate Src and eventually ERK-dependent signaling. One of the many consequences of ERK activation that might be relevant to PECAM-1-dependent filopodia formation could be the activation of myosin light chain kinase and the subsequent phosphorylation of the light chains of actin-associated myosins. ${ }^{49}$ Additionally, in processes that do not appear to directly involve SHP-2-mediated ERK signaling, and which are poorly understood, PECAM-1 is involved in the regulation of Cdc42 expression. Studies are underway to evaluate the validity of this model.

The involvement of PECAM-1 in pathological angiogenesis has raised the possibility of PECAM-1 as a future target for anti-cancer therapy. ${ }^{55}$ The appeal of PECAM-1 is further enhanced by the fact that loss of PECAM-1 does not cause a debilitating vascular phenotype ${ }^{42}$ and significant vascular-related side effects have not been observed in mice treated with anti-PECAM-1 antibodies. ${ }^{39-41}$ This suggests that anti-PECAM-1 therapy is likely to be well tolerated. However, the involvement of PECAM-1 in the trafficking of white cell $s^{35}$ and in providing resistance to endotoxic and apoptotic stresses $^{36,37}$ means that consideration must still be given to the potential for immunosuppression and/or increased sensitivity to vascular insults during anti-PECAM-1 therapy. Consequently, developing a full understanding of the role of PECAM-1 in mediating endothelial cell functions will be essential for exploiting the therapeutic potential of targeting PECAM-1.

\section{Acknowledgements}

This work was supported by grants from the Department of Defense (PR043482) 


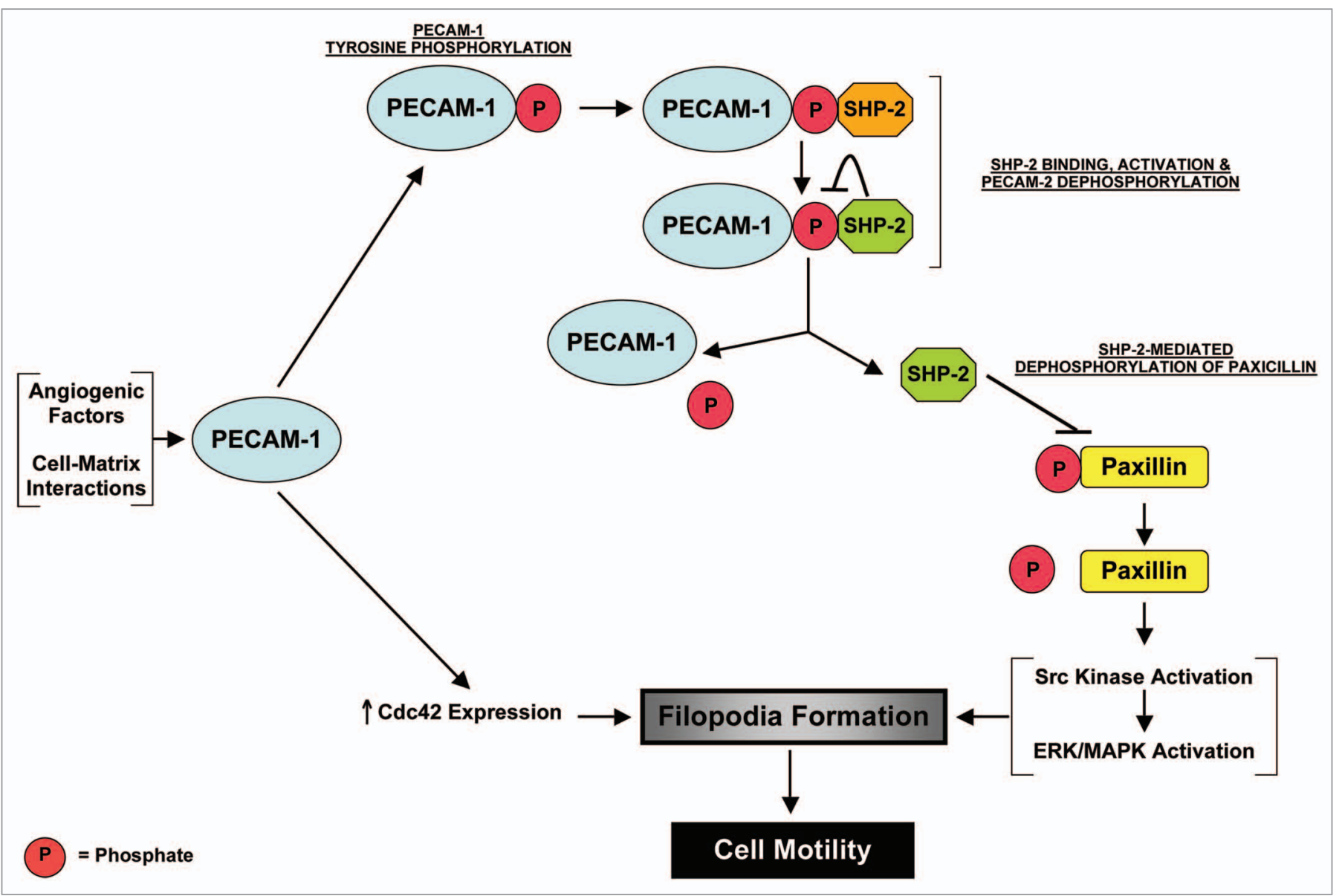

Figure 2. A proposed model for the involvement of PECAM-1 in the formation of endothelial cell filopodia during angiogenesis. During pathological or certain forms of developmental angiogenesis, angiogenic factors and/or cell-matrix interactions stimulate PECAM-1 tyrosine phosphorylation. These phosphorylation events lead to the binding of SHP-2 to PECAM-1 and the activation of SHP-2's phosphatase activity. The activated SHP-2 dephosphorylates the PECAM-1 molecule to which it is bound, leading to its release from PECAM-1. The liberated SHP-2 subsequently targets paxillin, dephosphorylating it, to trigger ERK-mediated activation of filopodia formation. Also, in processes that are still undefined, PECAM-1 may be involved in the regulation of $\mathrm{Cdc} 42$ during angiogenesis.

and the National Institutes of Health (HL079090).

\section{References}

1. Nambiar R, McConnell RE, Tyska MJ. Myosin motor function: the ins and outs of actin-based membrane protrusions. Cell Mol Life Sci 2010; 67: 1239-54.

2. Pollard TD, Borisy GG. Cellular motility driven by assembly and disassembly of actin filaments. Cell 2003; 112:453-65.

3. Small JV, Auinger S, Nemethova M, Koestler S, Goldie KN, Hoenger A, et al. Unravelling the structure of the lamellipodium. J Microsc 2008; 231:479-85.

4. Faix J, Rottner K. The making of filopodia. Curr Opin Cell Biol 2006; 18:18-25.

5. Mattila PK, Lappalainen P. Filopodia: molecular architecture and cellular functions. Nat Rev Mol Cell Biol 2008; 9:446-54.

6. Ferrara N, Kerbel RS. Angiogenesis as a therapeutic target. Nature 2005; 438:967-74.

7. Adams RH, Alitalo K. Molecular regulation of angiogenesis and lymphangiogenesis. Nat Rev Mol Cell Biol 2007; 8:464-78.
8. De Smet F, Segura I, De Bock K, Hohensinner PJ, Carmeliet P. Mechanisms of vessel branching: filopodia on endothelial tip cells lead the way. Arterioscler Thromb Vasc Biol 2009; 29:639-49.

9. Gerhardt $H$, Golding $M$, Fruttiger $M$, Ruhrberg C, Lundkvist A, Abramsson A, et al. VEGF guides angiogenic sprouting utilizing endothelial tip cell filopodia. J Cell Biol 2003; 161:1163-77.

10. Gerhardt H, Ruhrberg C, Abramsson A, Fujisawa H, Shima D, Betsholtz C. Neuropilin-1 is required for endothelial tip cell guidance in the developing central nervous system. Dev Dyn 2004; 231:503-9.

11. Cao G, Fehrenbach M, Williams J, Finklestein J, Zhu JX, DeLisser HM. Angiogenesis in PECAM-1-null mice. Am J Path 2009; 175:903-15.

12. Zhu JX, Cao G, Fehrenbach M, Williams J, DeLisser HM. SHP-2 phosphatase activity is required for PECAM-1-dependent cell motility. Am J Physiol Cell Physiol 2010;299:C854-65.

13. Ilan N, Madri JA. PECAM-1: old friend, new partners. Curr Opin Cell Biol 2003; 15:515-24.

14. Jackson DE. The unfolding tale of PECAM-1. FEBS Lett 2003; 540:7-14.

15. Newman PJ, Newman DK. Signal transduction pathways mediated by PECAM-1: new roles for an old molecule in platelet and vascular cell biology. Arterioscler Thromb Vasc Biol 2003; 23:953-64
16. Fujiwara K. Platelet endothelial cell adhesion molecule- 1 and mechanotransduction in vascular endothelial cells. J Intern Med 2006; 259:373-80.

17. Newman PJ, Berndt MC, Gorski J, White GC II, Lyman S, Paddock C, et al. PECAM-1(CD31) cloning and relation to adhesion molecules of the immunoglobulin gene superfamily. Science 1990; 247:1219-22.

18. Prager E, Sunder-Plassman R, Hansmann C, Koch C, Holter W, Knapp W, et al. Interaction of CD31 with a heterophilic counterreceptor involved in downregulation of human $\mathrm{T}$ cell responses. J Exp Med 1996; 184:41-50.

19. DeLisser HM, Yan HC, Newman PJ, Muller WA, Buck CA, Albelda SM. Platelet/endothelial cell adhesion molecule-1 (CD31)-mediated cellular aggregation involves cell surface glycosaminoglycans. J Biol Chem 1993; 268:16037-46.

20. Sun J, Williams J, Yan HC, Amin KM, Albelda SM DeLisser HM. Platelet endothelial cell adhesion molecule-1 (PECAM-1) homophilic adhesion is mediated by immunoglobulin-like domains 1 and 2 and depends on the cytoplasmic domain and the level of surface expression. J Biol Chem 1996 271:18561-70. 
21. Coombe DR, Stevenson SM, Kinnear BF, Gandhi NS, Mancera RL, Osmond RI, et al. Platelet endothelial cell adhesion molecule 1 (PECAM-1) and its interactions with glycosaminoglycans: 2 . Biochemical analyses. Biochemistry 2008; 47:4863-75.

22. Sachs UJ, Andrei-Selmer CL, Maniar A, Weiss T, Paddock C, Orlova VV, et al. The neutrophil-specific antigen CD177 is a counter-receptor for platelet endothelial cell adhesion molecule-1 (CD31). J Biol Chem 2007; 282:23603-12.

23. Billadeau DD, Leibson PJ. ITAMs versus ITIMs: striking a balance during cell regulation. J Clin Invest 2002; 109:161-8.

24. Jackson DE, Kupcho KR, Newman PJ. Characterization of phosphotyrosine binding motifs in the cytoplasmic domain of platelet/endothelial cell adhesion molecule-1 (PECAM-1) that are required for the cellular association and activation of the protein-tyrosine phosphatase, SHP-2. J Biol Chem 1997; 272:24868-75.

25. Cao MY, Huber M, Beauchemin N, Famiglietti J, Albelda SM, Veillette A. Regulation of mouse PECAM-1 tyrosine phosphorylation by the Src and Csk families of protein-tyrosine kinases. J Biol Chem 1998; 273:15765-72.

26. Sagawa K, Kimura T, Swieter M, Siraganian RP. The protein-tyrosine phosphatase SHP-2 associates with tyrosine-phosphylated adhesion molecule PECAM-1 (CD31). J Biol Chem 1997; 272:31086-91.

27. Masuda M, Osawa M, Shigematsu H, Harada N, Fujiwara K. Platelet endothelial cell adhesion molecule-1 is a major SH-PTP2 binding protein in vascular endothelial cells. FEBS Lett 1997; 408:331-6.

28. Jackson DE, Ward CM, Wang R, Newman PJ. The protein-tyrosine phosphatase SHP-2 binds platelet/ endothelial cell adhesion molecule-1 (PECAM-1) and forms a distinct signaling complex during platelet aggregation. J Biol Chem 1997; 272:6986-93.

29. Hua CT, Gamble JR, Vadas MA, Jackson DE. Recruitment and activation of SHP-1 protein-tyrosine phosphatase by human platelet endothelial cell adhesion molecule-1 (PECAM-1). J Biol Chem 1998; 273:28332-40.

30. Edmead CE, Crosby DA, Southcott M, Poole AW. Thrombin-induced association of SHP-2 with multiple tyrosine-phosphorylated proteins in human platelets. FEBS Lett 1999; 459:27-32.

31. Pumphrey NJ, Taylor V, Freeman S, Douglas MR, Bradfield PF, Young SP, et al. Differential association of cytoplasmic signalling molecules SHP-1, SHP-2, SHIP and phospholipase C- $\gamma 1$ with PECAM-1/ CD31. FEBS Lett 1999; 450:77-83.
32. Pellegatta F, Chierchia SL, Zocchi MR. Functional association of platelet endothelial cell adhesion molecule-1 and phosphoinositide 3-kinase in human neutrophils. J Biol Chem 1998; 273:27768-77.

33. Ilan N, Mahooti S, Rimm DL, Madri JA. PECAM-1 (CD31) functions as a reservoir for and a modulator of tyrosine-phosphorylated $\beta$-catenin. J Cell Sci 1999; 112:3005-14

34. Ilan N, Cheung L, Pinter E, Madri JA. Plateletendothelial cell adhesion molecule-1 (CD31), a scaffolding molecule for selected catenin family members whose binding is mediated by different tyrosine and serine/threonine phosphorylation. J Biol Chem 2000; 275:21435-43.

35. Woodfin A, Voisin MB, Nourshargh S. PECAM-1: a multi-functional molecule in inflammation and vascular biology. Arterioscler Thromb Vasc Biol 2007; 27:2514-23.

36. Carrithers M, Tandon S, Canosa S, Michaud M, Graesser D, Madri JA. Enhanced susceptibility to endotoxic shock and impaired STAT3 signaling in CD31-deficient mice. Am J Pathol 2005; 166:185-96.

37. Maas M, Stapleton M, Bergom C, Mattson DL, Newman DK, Newman PJ. Endothelial cell PECAM1 confers protection against endotoxic shock. Am J Physiol Heart Circ Physiol 2005; 288:159-64.

38. Tzima E, Irani-Tehrani M, Kiosses WB, Dejana E, Schultz DA, Engelhardt B, et al. A mechanosensory complex that mediates the endothelial cell response to fluid shear stress. Nature 2005; 437:426-31.

39. DeLisser HM, Christofidou-Solomidou M, Strieter RM, Burdick MD, Robinson CS, Wexler RS, et al. Involvement of endothelial PECAM-1/CD31 in angiogenesis. Am J Pathol 1997; 151:671-7.

40. Cao G, O'Brien CD, Zhou Z, Sanders SM, Greenbaum JN, Makrigiannakis A, et al. The involvement of human PECAM-1 in angiogenesis and in vitro endothelial cell migration. Am J Physiol Cell Physiol 2002; 282:1181-90.

41. Zhou Z, Christofidou-Solomidou M, Garlanda C, DeLisser HM. Antibody against murine PECAM-1 inhibits tumor angiogenesis in mice. Angiogenesis 1999; 3:181-8.

42. Duncan GS, Andrew DP, Takimoto H, Kaufman SA, Yoshida H, Spellberg J, et al. Genetic evidence for functional redundancy of Platelet/Endothelial cell adhesion molecule-1 (PECAM-1): CD31 deficient mice reveal PECAM-1-dependent and PECAM-1-independent functions. J Immunol 1999; 162:3022-30.
43. Solowiej A, Biswas P, Graesser D, Madri JA. Lack of platelet endothelial cell adhesion molecule-1 attenuates foreign body inflammation because of decreased angiogenesis. Am J Pathol 2003; 162:953-62.

44. DeLisser HM, Helmke BP, Cao G, Egan PM, Taichman D, Fehrenbach M, et al. Loss of PECAM-1 function impairs alveolarization. J Biol Chem 2006; 281:8724-31.

45. Gratzinger D, Canosa S, Engelhardt B, Madri JA Platelet endothelial cell adhesion molecule-1 modulates endothelial cell motility through the small G-protein Rho. FASEB J 2003; 17:1458-69.

46. Kondo S, Scheef EA, Sheibani N, Sorenson CM. PECAM-1 isoform-specific regulation of kidney endothelial cell migration and capillary morphogenesis. Am J Physiol Cell Physiol 2007; 292:2070-83.

47. O'Brien C, Cao G, Makringiannakis A, DeLisser HM. The role of immunoreceptor tyrosine-based inhibitory motifs of platelet endothelial cell adhesion molecule (PECAM-1) in PECAM-1 dependen cell migration. Am J Physiol Cell Physiol 2004; 287:1103-13.

48. Brahmbhatt AA, Klemke RL. ERK and RhoA differentially regulate pseudopodia growth and retraction during chemotaxis. J Biol Chem 2003; 278:13016-25.

49. Huang C, Jacobson K, Schaller MD. MAP kinase and cell migration. J Cell Sci 2004; 117:4619-28.

50. Ren Y, Meng S, Mei L, Zhao ZJ, Jove R, Wu J. Roles of Gab1 and SHP2 in paxillin tyrosine dephosphorylation and Src activation in response to epidermal growth factor. J Biol Chem 2005; 279:8497-505.

51. Dance M, Montagner A, Salles JP, Yart A, Raynal P. The molecular functions of Shp2 in the Ras/ Mitogen-activated protein kinase (ERK1/2) pathway. Cell Signal 2008; 20:453-9.

52. Raftopoulou M, Hall A. Cell migration: Rho GTPases lead the way. Dev Biol 2004; 265:23-32.

53. Cerione RA. Cdc42: new roads to travel. Trends Cell Biol 2004; 14:127-32.

54. Yang L, Wang L, Zheng Y. Gene targeting of Cdc42 and Cdc42GAP affirms the critical involvement of Cdc42 in filopodia induction, directed migration and proliferation in primary mouse embryonic fibroblasts. Mol Biol Cell 2006; 17:4675-85.

55. DeLisser HM. Targeting PECAM-1 for anti-cancer therapy. Canc Biol Ther 2007; 6:121-2. 